\title{
Theoretical investigation of thermal effects in non-isothermal non-equilibrium reactive liquid chromatography
}

\author{
Shamsul Qamar ${ }^{\mathrm{a}, \mathrm{b}, *}$, Fouzia A. Sattar ${ }^{\mathrm{a}}$, Andreas Seidel-Morgenstern ${ }^{\mathrm{b}}$ \\ ${ }^{a}$ Department of Mathematics, COMSATS Institute of Information Technology, Islamabad, Pakistan \\ ${ }^{b}$ Max Planck Institute for Dynamics of Complex Technical Systems Magdeburg, Germany
}

\begin{abstract}
A mathematical model is formulated and numerically approximated to simulate reaction and separation occurring jointly in a chromatographic column. To cover realistic problems, the reversibility of the reactions and the occurrence of temperature gradients are considered. The model is formed by a system of convection-diffusion-reaction partial differential equations coupled with differential and algebraic equations. The presence of nonlinear transport dominated terms in mass and energy balance equations and stiffness of the reaction terms are the main sources of instabilities if simple numerical schemes are applied. In this work a high resolution finite volume scheme is applied to accurately solve the model equations. The numerical case studies, treating two stoichiometrically different reactions, demonstrate the degree of coupling concentration and thermal fronts. The impact of several key parameters on process performance is illustrated. The results obtained are seen as very useful to understand the velocities and shapes of concentration and thermal fronts in chromatographic reactors. They reveal potential for improving reactor performance exploiting the unavoidable non-isothermal operation.
\end{abstract}

Keywords: Chromatographic reactor, non-isothermal process, non-equilibrium model, thermal effects, finite volume scheme.

\footnotetext{
*Corresponding author. Tel: +92 51 8318471; fax: +92 518318499

Email address: shamsul.qamar@comsats.edu.pk (Shamsul Qamar)
} 


\section{Introduction}

Fixed-bed absorbers or desorbers have high demand in various process industries where traditional separation operations like distillation, solvent extraction, crystallization and evaporation are not applicable due to physicochemical limitations. In chromatographic reactors, chemical or biochemical reactions and chromatographic separations take place simultaneously. The setup provides an integrated process in a single unit that enhances the conversion of reactants and product purity. In contrast to the sequentially connected conventional reactors and separators, chromatographic reactors effectively reduces the number of units and improves the conversion, yield and separation capacity. During the elution of reactants pulses through a chromatographic reactor, heats are generated or consumed continuously due to enthalpies of adsorption, chemical reaction, and mixing. Additionally, thermal effects can also originate from viscous heat dissipation, particularly in HPLC columns packed with fine particles, see Brandt et al. (1997). Such phenomena should be taken into account in scale-up of the reactor concept because increasing the column diameter eventually renders the system nearly adiabatic.

The concept of a fixed-bed chromatographic reactor can be better explained by considering a single chromatographic column in which a reversible reaction of the type $A \rightleftarrows B+C$ takes place. In this model process, rectangular pulses of reactant $A$ are periodically injected into an inert carrier stream that elutes through the column packed with the stationary phase. During the elution, the reactant $A$ reacts to form the products $B$ and $C$ due to the catalytic effects of the solid phase. Because of different affinities of the components $B$ and $C$, they move at different velocities in the chromatographic reactor. Resultantly, both components are separated and their backward reaction is suppressed, leading to high conversion at the column outlet. The situation is highly favorable, when the reactant $A$ elutes between the products $B$ and $C$. A complete conversion of the reactant is possible when the residence time of the sample in the reactor is long enough. For further details about the principles and applications of chromatographic reactors see Villermaux (1981); Ganetsos (1993); Sardin (1993); Borren and Fricke (2005); Fricke (2005). 
Temperature distributions are typically neglected in the analysis of liquid chromatography and chromatographic reactors by assuming that the effects caused by heats of adsorption and reaction are negligible. For that reason most of the available literature is limited to the isothermal conditions. Only a few contributions considering non-isothermal conditions are available in the literature, see e.g. Sainio (2005, 2007, 2011); Tien and Seidel-Morgenstern (2011); Graça et al. (2012a,b); Javeed et al. (2012). On the other hand, thermal effects have been widely discussed in the case of gas phase reactions in solid packings, see e.g. Kruglov (1994); Yongsunthon (1999); Xiu (2002); Glöckler (2006); Eigenberger (2007).

Mathematical modeling is an essential part of the chromatographic theory for describing its dynamical process. It provides a procedure for predicting the dynamical behavior of solute in the column without extensive experiments. Due to different considerations of simplifications, several types of models have been established and applied to illustrate the behavior of chromatographic columns. These include the general rate model, the lumped kinetic model, the linear driving force model, the linear model, and the equilibrium dispersive model, see e.g. Ruthven (1984); Carta (1988); Guiochon (2002); Guiochon and Lin (2003); Guiochon et al. (2006). All these models need an important input information regarding the thermodynamic equilibrium of the distribution of the components between the mobile and stationary phases. These models are categorized as non-equilibrium and equilibrium transport models. In the equilibrium models sorption is presumed to be instantaneous, while sorption in the non-equilibrium models is thought to be governed by first-order kinetics, see Ruthven (1984); Guiochon (2002); Guiochon et al. (2006).

The purpose of this contribution is to quantify how thermal effects affect conversion and separation in non-isothermal and non-equilibrium liquid phase chromatographic reactors. When the heat of reaction and enthalpy of adsorption are sufficiently high, the system may deviate significantly from isothermal behavior. In such a scenario, it is necessary to include a partial differential equation (PDE) for the energy balance in the existing model equations of isothermal chromatographic reactor. A high resolution finite volume scheme is proposed to solve the model equations. Several case studies of three and four-component reactions are presented to illustrate the interactions of concentration and thermal fronts 
and to identify the key parameters that influence the reactor performance. The results

demonstrate that the reactor performance considerably improves under non-isothermal operation as compared to isothermal operation, i.e. both conversion of reactants and the amount products are increased.

This article is arranged as follows. In Section 2, a non-equilibrium mathematical model for non-isothermal liquid chromatographic reactor is formulated. In Section 3, a high resolution flux-limiting finite volume scheme is derived and implemented for solving the model equations. Section 4 introduces a procedure for some tests to check the consistency of results. In Section 5, numerical case studies are carried out. Finally, Section 6 gives conclusions and remarks.

\section{The mathematical model}

This section presents a mathematical model for describing the non-equilibrium transport of reactants and products in a single-column adiabatic chromatographic reactor. Mathematically, the non-isothermal single-column chromatographic reactor is analogous to a more frequently studied non-isothermal unsteady-state fixed-bed reactor, see e.g. Rhee et al. (1989); Sainio (2007).

In this work, the nonlinear reactive lumped kinetic model (RLKM) is used to describe the non-equilibrium and non-isothermal transport of reactants and products in an adiabatic chromatographic reactor. The model incorporates the rate of variation of the local concentration of solute in the stationary phase and back-mixing in the column due to dispersion. It lumps hereby the contribution of internal and external mass transport resistances into a mass transfer coefficient denoted by $k$. It is assumed that a single reaction takes place exclusively in the solid phase. There are no radial concentration and temperature gradients in the column and sample migrates in the $z$-direction by advection and axial-dispersion. Thus, only axial-dispersions cause band broadening denoted by $D_{z, i}$ and $\lambda_{z}$ in the mass and energy balances, respectively. Compressibility of the mobile phase is assumed to be negligible. No interactions take place between the solvent (carrier) and the solid phase. 
Moreover, there is no heat added or removed from the system except via the inlet and outlet streams, i.e. reactor is operated under adiabatic condition.

Based on the above assumptions, the mass equations of a one-dimensional RLKM for a fixed-bed chromatographic reactor can be expressed as (e.g. Zhong and Meunier (1994); Guiochon et al. (2006))

$$
\begin{gathered}
\frac{\partial c_{i}}{\partial t}=-u \frac{\partial c_{i}}{\partial z}+D_{z, i} \frac{\partial^{2} c_{i}}{\partial z^{2}}-F k_{i}\left(q_{i}^{*}-q_{i}\right), \quad i=1,2, \ldots, N_{c} \\
\frac{\partial q_{i}}{\partial t}=k_{i}\left(q_{i}^{*}-q_{i}\right)+\nu_{i} r^{\text {het }}
\end{gathered}
$$

Here, $c_{i}$ is the concentration of the $i$-th component in the fluid phase, $q_{i}$ is the solid phase equilibrium concentration of the $i$-th component, $u$ represents the constant interstitial velocity of the mobile phase, $D_{z, i}$ denotes the axial-dispersion coefficient of $i$-th component, $t$ is the time, and $z$ is the axial-coordinate along the column. Moreover, $F=(1-\epsilon) / \epsilon$ is the phase ratio based on the porosity $\epsilon \in(0,1), r^{\text {het }}$ is the heterogeneous reaction rate, $\nu_{i}$ is the corresponding stoichiometric coefficient of $i$-th component, and $N_{c}$ represents the total number of components. Note that, the stoichiometric coefficient $\nu_{i}$ is negative for reactants and positive for products. For large values of the mass transfer coefficient $k$, i.e. for $k \rightarrow \infty$, the current non-equilibrium RLKM reduces to the reactive equilibrium dispersive model (REDM) studies by Javeed et al. (2012).

If the enthalpy of mixing is neglected, the non-equilibrium energy balances for a differential volume element in an adiabatic chromatographic reactor are given as (e.g. Zhong and Meunier (1994); Guiochon et al. (2006))

$$
\begin{gathered}
\rho^{L} c_{p}^{L} \frac{\partial T}{\partial t}=-u \rho^{L} c_{p}^{L} \frac{\partial T}{\partial z}+\lambda_{z} \frac{\partial^{2} T}{\partial z^{2}}-F \frac{3 h_{p}}{R_{p}}\left(T-T_{s}\right), \\
\rho^{S} c_{p}^{S} \frac{\partial T_{s}}{\partial t}=\sum_{l=1}^{N_{c}}\left(-\Delta H_{A, l}\right) \frac{\partial q_{l}}{\partial t}+\frac{3 h_{p}}{R_{p}}\left(T-T_{s}\right)+\left(-\Delta H_{R}\right) r^{\text {het }} .
\end{gathered}
$$

In the above equations, $T$ denotes temperature of the liquid phase, $T_{s}$ is temperature of the solid phase, $\rho$ is the density per unit volume, $\lambda_{z}$ is the axial heat conductivity coefficient, 
$c_{\mathrm{p}}$ is the heat capacity, and the superscripts $L$ and $S$ denote the liquid and solid phases, respectively. The considered density and heat capacity are not depending on temperature and composition. Moreover, $\Delta H_{\mathrm{A}, \mathrm{i}}$ is the enthalpy of adsorption for the $i$-th component, $\Delta H_{\mathrm{R}}$ is the enthalpy of reaction, $h_{p}$ is the heat transfer coefficient quantifying the rate of the heat exchange between the mobile and stationary phases, and $R_{p}$ is the radius of solid particles.

The equilibrium adsorption isotherms are assumed to be linear in term of the concentrations and nonlinear in term of the temperature as given below:

$$
q_{i}^{*}=a_{i}\left(T_{s}\right) c_{i}
$$

where

$$
a_{i}\left(T_{s}\right)=a_{i}^{\mathrm{ref}} \exp \left(\frac{-\Delta H_{A, i}}{R_{g}}\left(\frac{1}{T_{s}}-\frac{1}{T^{\mathrm{ref}}}\right)\right) .
$$

In the above equations $a_{i}^{\text {ref }}$ represents the Henry's constant for $i$-th component at reference temperature $T^{\mathrm{ref}}$ and $R_{g}$ denotes the general gas constant. Based on the conservation laws, the reaction rate for the considered three-component model reaction is expressed as

$$
r^{\text {het }}=k^{\text {het }}\left(T_{s}\right)\left(q_{A}-\frac{q_{B} q_{C}}{K_{\mathrm{eq}}^{\text {het }}\left(T_{s}\right)}\right) .
$$

Here, $k^{\text {het }}$ and $K_{e q}^{\text {het }}$ denote the heterogeneous forward reaction rate and reaction equilibrium constants, respectively. The effect of temperature on the chemical reaction rate $k^{\text {het }}\left(T_{s}\right)$ is an exponential function of the absolute temperature as described by the Arrhenius equation using the activation energy $E_{A}$ :

$$
k^{\text {het }}\left(T_{s}\right)=k^{\text {het }}\left(T^{\mathrm{ref}}\right) \exp \left(\frac{-E_{A}}{R_{g}}\left(\frac{1}{T_{s}}-\frac{1}{T^{\mathrm{ref}}}\right)\right) .
$$

Here, $E_{A}$ denotes the activation energy. The reaction equilibrium constant depends on the temperature as given below

$$
K_{\mathrm{eq}}^{\mathrm{het}}\left(T_{s}\right)=K_{\mathrm{eq}}^{\mathrm{het}}\left(T^{\mathrm{ref}}\right) \exp \left(\frac{-\Delta H_{R}}{R_{g}}\left(\frac{1}{T_{s}}-\frac{1}{T^{\mathrm{ref}}}\right)\right) .
$$

Eqs. (1)-(4) are solved using the appropriate initial and boundary conditions. 


\section{Initial Conditions:}

$$
c_{i}(z, 0)=c_{i}^{\mathrm{init}}, \quad T(z, 0)=T^{\mathrm{ref}}, q_{i}(z, 0)=q_{i}^{*}(z, 0), \quad T_{s}(z, 0)=T^{\mathrm{ref}}, i=1,2, \cdots, N_{c}
$$

where, $c_{i}^{\text {init }}$ is the equilibrated initial concentration of $i$-th component and $T^{\text {ref }}$ is the reference temperature.

Boundary Conditions: The standard Danckwerts boundary conditions are applied at the column inlet and outlet for both concentrations and temperature:

$$
\begin{gathered}
-\frac{D_{z, i}}{L u} \frac{\partial c_{i}}{\partial z}+\left.c_{i}\right|_{z=0}=\left\{\begin{array}{l}
c_{i}^{\mathrm{inj}}, \quad 0 \leq t \leq t_{\mathrm{inj}} \\
0, \quad t>t_{\mathrm{inj}}
\end{array},\left.\quad \frac{\partial c_{i}}{\partial z}\right|_{z=L}=0,\right. \\
-\frac{\lambda_{a z}}{L u \rho^{L} C_{p}^{L}} \frac{\partial T}{\partial z}+\left.T\right|_{z=0}=\left\{\begin{array}{ll}
T^{\mathrm{inj}}, & 0 \leq t \leq t_{\mathrm{inj}} \\
T^{\mathrm{ref}}, & t>t_{\mathrm{inj}}
\end{array},\left.\quad \frac{\partial T}{\partial z}\right|_{z=L}=0,\right.
\end{gathered}
$$

where, $c_{i}^{\text {inj }}$ is the injected concentration of $i$-th component and $T^{\text {inj }}$ is the sample temperature at injection. The formulation of the thermal inlet boundary conditions in Eq. (11b) allows the analysis of interesting cases, in which the sample is injected with a temperature, $T_{\mathrm{inj}}$, differing from the inlet temperature of the mobile phase, $T_{\text {ref }}$.

This completes the derivation of the model for the non-equilibrium and non-isothermal chromatographic reactor. In the next section, the suggested flux-limiting HR-FVS is applied to solve the model equations.

\section{Numerical scheme}

In this section, the high resolution finite volume scheme of Koren (1993) is extended to solve the model equations given by Eqs. (1) and (3). For simplicity and better explanation of the scheme, we consider a three-component reaction. Then, Eqs. (1) and (3) can be re-written as

$$
\frac{\partial \mathbf{w}}{\partial t}+\mathbf{U} \frac{\partial \mathbf{w}}{\partial z}=\mathbf{D} \frac{\partial^{2} \mathbf{w}}{\partial z^{2}}+\mathbf{Q}
$$

where 


$$
\begin{aligned}
\mathbf{w} & =\left(\begin{array}{l}
c_{1} \\
c_{2} \\
c_{3} \\
T
\end{array}\right), \quad \mathbf{U}=\left(\begin{array}{llll}
u & 0 & 0 & 0 \\
0 & u & 0 & 0 \\
0 & 0 & u & 0 \\
0 & 0 & 0 & u
\end{array}\right), \quad \mathbf{D}=\left(\begin{array}{cccc}
D_{z, 1} & 0 & 0 & 0 \\
0 & D_{z, 2} & 0 & 0 \\
0 & 0 & D_{z, 3} & 0 \\
0 & 0 & 0 & \frac{\lambda_{z}}{\rho^{L} c_{p}^{L}}
\end{array}\right) \\
\mathbf{Q} & =\left(\begin{array}{c}
-F k_{1}\left(q_{1}^{*}-q_{1}\right) \\
-F k_{2}\left(q_{2}^{*}-q_{2}\right) \\
-F k_{3}\left(q_{3}^{*}-q_{3}\right) \\
-F \frac{3 h_{p}}{R_{p} \rho^{L} c_{P}^{L}}\left(T-T_{s}\right)
\end{array}\right) .
\end{aligned}
$$

Before applying the proposed numerical scheme to Eq. (12), it is required to discretize the computational domain. Let $N_{z}$ represent the number of discretization points and the points $\left(z_{j-\frac{1}{2}}\right), j \in\left\{1, \cdots, N_{z}+1\right\}$ be partitions of the interval $[0,1]$. For each $j=1,2, \cdots, N_{z}$, $\Delta z$ is a constant width of each mesh interval. The $z_{j}$ denote the cell centers, and $z_{j \pm \frac{1}{2}}$ refer to the cell boundaries. We assign

$$
z_{1 / 2}=0, \quad z_{N+1 / 2}=1, \quad z_{j+1 / 2}=j \cdot \Delta z, \text { for } j=1,2, \cdots N_{z} .
$$

Moreover, we have

$$
z_{j}=\left(z_{j-1 / 2}+z_{j+1 / 2}\right) / 2 \quad \text { and } \quad \Delta z=z_{j+1 / 2}-z_{j-1 / 2}=\frac{1}{N_{z}+1} .
$$

Let $\Omega_{j}:=\left[z_{j-1 / 2}, z_{j+1 / 2}\right]$ for $j \geq 1$. The cell averaged initial data in each cell are given as

$$
\mathbf{w}_{j}(0)=\frac{1}{\Delta z} \int_{\Omega_{j}} \mathbf{w}^{\text {init }}(z) d z, \text { for } j=1,2, \cdots N_{z}
$$

By integrating Eq. (12a) over the interval $\Omega_{j}$, we obtain

$$
\int_{\Omega_{j}} \frac{\partial \mathbf{w}}{\partial t} d z=-\mathbf{U}\left(\mathbf{w}_{j+\frac{1}{2}}-\mathbf{w}_{j-\frac{1}{2}}\right)+\mathbf{D}\left[\left(\frac{\partial \mathbf{w}}{\partial z}\right)_{j+1 / 2}-\left(\frac{\partial \mathbf{w}}{\partial z}\right)_{j-1 / 2}\right]+\int_{\Omega_{j}} \mathbf{Q} d z .
$$

In each $\Omega_{j}$, the averaged values of the conservative variables are given as

$$
\mathbf{w}_{j}(t)=\frac{1}{\Delta z} \int_{\Omega_{j}} \mathbf{w}(t, z) d z .
$$


In Eq. (17), the vector $\mathbf{w}_{j}$ contains the cell averaged conservative variables, while in Eq. (16) the vector $\mathbf{w}_{j \pm \frac{1}{2}}$ contains conservative variables at the respective cell boundaries. Similarly, the cell-averaged value of source term $\mathbf{Q}$ can be defined. Therefore, by using Eq. (17) in Eq. (16), the following semi-discrete scheme is obtained, for each, $j=1,2, \cdots, N_{z}$,

$$
\frac{d \mathbf{w}_{j}}{d t}=-\frac{\mathbf{U}}{\Delta z}\left(\mathbf{w}_{j+\frac{1}{2}}-\mathbf{w}_{j-\frac{1}{2}}\right)+\frac{\mathbf{D}}{\Delta z}\left[\left(\frac{\partial \mathbf{w}}{\partial z}\right)_{j+1 / 2}-\left(\frac{\partial \mathbf{w}}{\partial z}\right)_{j-1 / 2}\right]+\mathbf{Q}_{j} .
$$

The differential terms of the diffusion part can be approximated as

$$
\left(\frac{\partial \mathbf{w}}{\partial z}\right)_{j \pm \frac{1}{2}}= \pm\left(\frac{\mathbf{w}_{j \pm 1}-\mathbf{w}_{j}}{\Delta z}\right)
$$

The next step is to approximate the values for the convective variables at the cell interfaces $\mathbf{w}_{j \pm \frac{1}{2}}$ in Eq. (18). In this study, a flux-limiting high resolution scheme of Koren is considered, see Koren (1993); Javeed et al. (2011). This scheme uses the Sweby-type fluxlimiter (c.f. Sweby (1984)) to preserve the local monotonicity of the scheme. According to this scheme the components of $\mathbf{w}$ at the cell interfaces, i.e. $\left(w_{i}\right)_{j+\frac{1}{2}}$ for $i=1,2,3,4$, are approximated as

$$
\left(w_{i}\right)_{j+\frac{1}{2}}=\left(w_{i}\right)_{j}+\frac{1}{2} \phi\left(\left(r_{i}\right)_{j+\frac{1}{2}}\right)\left(\left(w_{i}\right)_{j}-\left(w_{i}\right)_{j-1}\right)
$$

where $\left(r_{i}\right)_{j+\frac{1}{2}}$ are the ratios of gradients

$$
\left(r_{i}\right)_{j+\frac{1}{2}}=\frac{\left(w_{i}\right)_{j+1}-\left(w_{i}\right)_{j}+\zeta}{\left(w_{i}\right)_{j}-\left(w_{i}\right)_{j-1}+\zeta} .
$$

Similarly, $\left(w_{i}\right)_{j-\frac{1}{2}}$ can be approximated by lowering the index $j$ by one. Here $\zeta \approx 10^{-10}$ is used to avoid division by zero and the limiting function $\phi$ is given as

$$
\phi\left(\left(r_{i}\right)_{j+\frac{1}{2}}\right)=\max \left(0, \min \left(2\left(r_{i}\right)_{j+\frac{1}{2}}, \min \left(\frac{1}{3}+\frac{2}{3}\left(r_{i}\right)_{j+\frac{1}{2}}, 2\right)\right)\right) .
$$

Due to flux-limiting, the above scheme is a second order accurate, see Koren (1993); Javeed et al. (2011).

The approximation in Eq. (20) is not applicable to the boundary intervals. Let us specifically consider the left boundary related to the inflow boundary condition. The position of 
the interval face $z_{\frac{1}{2}}$ and inflow boundary are identical. However, $z_{0}$ is not known, therefore, Eq. (20) is not applicable at $z_{\frac{3}{2}}$. To overcome this problem, the first order backward difference scheme can be used at the cell interfaces $z_{\frac{3}{2}}$ and $z_{N_{z}+\frac{1}{2}}$. Let $\left(w_{i}\right)^{\text {inj }}$ represent the concentration of $i$-th injected pulse, then

$$
\left(w_{i}\right)_{\frac{1}{2}}=\left(w_{i}\right)^{\mathrm{inj}}, \quad\left(w_{i}\right)_{\frac{3}{2}}=\left(w_{i}\right)_{1}, \quad\left(w_{i}\right)_{N_{z}+\frac{1}{2}}=\left(w_{i}\right)_{N_{z}}, \quad i=1,2,3,4
$$

The above equation shows that outflow boundary conditions are used at the outlet of the column. The fluxes at other cell interfaces can be computed using Eq. (20). However, the use of a first order scheme in the boundary intervals does not affect the global accuracy of the method due to shrinking cell sizes with increasing node numbers. The efficiency and accuracy of the scheme were thoroughly analyzed in our previous article on nonlinear dispersive liquid chromatography, see Javeed et al. (2011).

The ODEs in Eqs. (2), (4) and Eq. (18) can be solved by a standard ODE-solver. In this

work, the numerical scheme was programmed in Matlab software and the resulting ODEs were solved by using the built-in Matlab routine ode23.

\section{Tests to check consistency of results}

The following integral consistency tests for mass and energy balances are helpful tools to verify the correctness of numerical results and the model equations. Here, these tests are performed to validate the accuracy of the numerical algorithm and the conservativity of mass and energy balances as illustrated below for the considered three-component $\mathrm{A} \leftrightarrows$ $\mathrm{B}+\mathrm{C}$ model reaction.

\subsection{Identity of integrated extents of reaction}

It is important to ensure that the total amount of concentration injected to the column remains conserved during the simulation of considered reversible chemical reaction $\mathrm{A} \leftrightarrows$ $\mathrm{B}+\mathrm{C}$. Conversion is a useful quantity for analyzing reversible reactions, because reactants are not converted entirely. 
The change in mole numbers of a mixture component $n_{k}$ is related to the stoichiometry of the reaction. For instance, in $\mathrm{A} \leftrightarrows \mathrm{B}+\mathrm{C}$ reaction, the integrated extent of reaction $\xi$ should obey the following relation

$$
\xi=n_{A}^{\text {inj }}-n_{A}^{\text {out }}=n_{B}^{\text {out }}+n_{C}^{\text {out }}
$$

Here, $\xi$ denotes all changes occurred in mole numbers due to the chemical reaction and $n_{k}^{\text {inj }}=c_{k}^{\text {inj }} V^{\text {inj }}$ quantifies the number of injected moles into the column. In the present study, the inlet concentrations of products i.e., $c_{B}^{\text {inj }}$ and $c_{C}^{\text {inj }}$, are taken to be zero. The symbol $V^{\text {inj }}$ represents the injected volume at the column inlet during the injection time $t^{\text {inj }}$.

The following integral formula can be used to calculate mole numbers at the column outlet

$$
n_{k}^{\text {out }}=\dot{V} \int_{0}^{t^{*}} c_{k}(t, z=L) \mathrm{d} t, \quad k=A, B, C,
$$

where $\dot{V}$ stands for the volumetric flow rate. In this study, the trapezoidal rule is used to approximate the above integrals. Large simulation time is considered to bring the system back to the initial state (time $\left.t^{*}\right)$.

The three values of $\xi_{k}$ can be utilized to calculate the standard deviation as follows

$$
\sigma_{\xi, k}[\%]=100 \times \sqrt{\frac{\sum_{i=1}^{k}\left(\xi_{k}-\bar{\xi}\right)^{2}}{3}}, \quad k=A, B, C .
$$

Here, $\bar{\xi}$ denotes the average of three $\xi_{k}$ for $k=A, B, C$. To respect the mass balances, this standard deviation should tend to zero.

\subsection{Integrated energy balance considering the extent of reaction}

An energetic evaluation of the process can be performed by comparing the enthalpies entering $\left(\Delta H^{\mathrm{inj}}\right)$ and leaving $\left(\Delta H^{\text {out }}\right)$ the system. These enthalpies are defined as

$$
\Delta H^{\mathrm{inj}}=\rho^{\mathrm{L}} c_{p}^{\mathrm{L}} \dot{V} \int_{0}^{t^{*}}\left(T^{\mathrm{inj}}-T^{\mathrm{ref}}\right) \mathrm{d} t, \quad \Delta H^{\mathrm{out}}=\rho^{\mathrm{L}} c_{p}^{\mathrm{L}} \dot{V} \int_{0}^{t^{*}}\left(T(t, z=L)-T^{\mathrm{ref}}\right) \mathrm{d} t .
$$

For $T^{\text {inj }}=T^{\text {ref }}$ holds $\Delta H^{\text {inj }}=0$. In the case of a complete adsorption and desorption cycle $\left(t^{*}\right.$ sufficiently long), there will be no overall sorption effect and the following balance 
equation can be derived using exclusively the effect of reaction quantified by $\Delta H_{\mathrm{R}}$ and $\xi$, e.g. Eq. (26)

$$
\Delta H^{\text {out }}+\left(\Delta H_{\mathrm{R}}\right) \bar{\xi}=0
$$

The fulfillment of Eq. (28) is required as a proof of accurate numerical simulations. There are several sources of numerical errors, such as discretization errors, round off errors, and errors in the numerical integrations of the outlet profiles, etc. Due to these errors, the right hand side of Eq. (28), called here $\Delta H_{\text {err }}$, might not be exactly zero, i.e.

$$
\Delta H_{\mathrm{err}}=\Delta H^{\mathrm{out}}+\left(\Delta H_{\mathrm{R}}\right) \bar{\xi}
$$

The smaller the $\Delta H_{\text {err }}$ the better is the fulfillment of the joint integral mass and energy balances. One can expect larger errors in $\Delta H_{\text {err }}$ compared to errors in $\xi$ due to the accumulation of all possible errors in this more critical consistency check. A relative percentage error in this energy can be given as

$$
E_{\mathrm{H}}[\%]=100 \times\left|\frac{\Delta H_{\mathrm{err}}}{\Delta H_{\mathrm{R}} \bar{\xi}}\right| .
$$

\section{Numerical case studies}

In this section some numerical case studies are carried out to investigate the effect of temperature on the elution profiles of three and four-component reactive chromatography. In this study, the axial dispersion, enthalpy of adsorption and mass transfer coefficients are assumed the same for all components, i.e $D_{z, i}=D_{z}, \Delta H_{A, i}=\Delta H_{A}$ and $k_{i}=k$. In practice, these quantities may vary with respect to components. All the parameters used in Tables 1 and 3 for three and four-component reactions were used by Sainio (2007) and Tien and Seidel-Morgenstern (2011) in their experiments. They are within the ranges of parameters typically encountered in HPLC applications.

\subsection{Three-component model reaction $(A \rightleftarrows B+C)$}

This subsection analyzes the thermodynamics and chemical kinetics of three-component reversible reaction in a heterogeneous system. The standard parameters used in this test problem are given in Table 1 and the reaction rate is given by Eq. (7). 
Figure 1 analyzes the isothermal behavior of the process for two different values of the mass transfer coefficient, i.e. $k=0.6 \mathrm{~min}^{-1}$ and $k=1200 \mathrm{~min}^{-1}$. This case study can be used as a reference case to understand the non-isothermal behavior. Here, $\Delta H_{A}=$ $\Delta H_{R}=0 \mathrm{~kJ} / \mathrm{mol}$. The results show the appearance of product components $\mathrm{B}$ and $\mathrm{C}$. The reference values of the Henry's constants reveal significant but incomplete conversion and separation. As expected, no changes are observed in the temperature. It is observed that for small value of $k$ the process is still not in equilibrium and the diffusion phenomena dominates the convection process. Conversion and separation of components improves on increasing the magnitude of $k$.

Figure 2 refers to the case study when the affect of enthalpy of adsorption $\Delta H_{A}$ is considered, while the enthalpy of reaction $\Delta H_{R}=0 \mathrm{~kJ} / \mathrm{mol}$. The concentration and corresponding temperature profiles are compared for two different values of mass transfer coefficient, i.e. $k=0.6 \mathrm{~min}^{-1}$ and $k=1200 \mathrm{~min}^{-1}$. The results are simulated by considering $\Delta H_{A}=-60 \mathrm{~kJ} / \mathrm{mol}$. The peaks are sharp and high enough when $k$ is large.

Figure 3 quantifies the effect of $\Delta H_{R}$ on the concentration and temperature profiles for $\Delta H_{A}=0 \mathrm{~kJ} / \mathrm{mol}$ and for two different values of $k$. The results are simulated under the influence of exothermic reaction i.e. $\Delta H_{R}=-20 \mathrm{~kJ} / \mathrm{mol}$. It is observed that for small value of $k$ the process is still not in equilibrium and the diffusion phenomena dominates the convection process. An increase in the magnitude of $k$ provides a significant rise in the conversion and separation of components. The same trend is seen in the temperature profiles as well.

In Figure 4 the effects of both $\Delta H_{A}$ and $\Delta H_{R}$ are analyzed. It is evident from the figures that for $k=0.6 \mathrm{~min}^{-1}$ the conversion and, hence, the separation is still incomplete and the corresponding temperature profile is more diffusive. It appears that by increasing the mass transfer coefficient to $1200 \mathrm{~min}^{-1}$, the results of RLKM approaches to that of reactive equilibrium dispersive model (REDM).

For the complete description of the non-isothermal chromatographic processes, the effects of enthalpies of adsorption and reaction are quantitatively evaluated in Table 2 by applying the mass balance consistency tests described in the previous section. The joint errors of the 
integral mass and energy balances, expressed by the error $E_{\mathrm{H}}$ (Eq. (30)), are less than 1 $\%$ which verifies the correctness of numerical solutions. For isothermal case, the extents of reactions are independent of the components and the results for the reference Henry's constants reveal significant but incomplete conversion and separation. In the non-isothermal case, the magnitudes of $\Delta H_{A}$ and $\Delta H_{R}$ significantly effect the conversion and separation. As the magnitude of $\Delta H_{R}$ increases, the temperature increases to a maximum value. Thus, the forward reaction is enhanced and the reactant is further converted. On the other hand, the magnitude of enthalpy of adsorption has an opposite effect on the temperature rise and conversion. The adsorption equilibrium constants decreases on increasing temperature, depending on the individual adsorption enthalpies. For $\Delta H_{\mathrm{A}, \mathrm{k}}=0 \mathrm{~kJ} / \mathrm{mol}$, the equilibrium constant are not influenced by the temperature. Moreover, for larger value of the activation energy $E_{\mathrm{A}}$ the reaction rate enhances due to an increase in the temperature. Thus, more reactant is converted into products with an increase in $E_{\mathrm{A}}$ value.

The elution order is an important factor in the performance of chromatographic reactor, as all reactants should be well separated to get the pure product. In the above test cases, the reactant was eluted in the middle of products, see Figures 2-4. In such a situation, the products can be obtained with high purity because both products are well separated which diminishes the backward reaction. Figure 5 demonstrates that how different permutations of adsorption coefficients significantly affect the conversion and separation of components in non-isothermal reactive chromatography. If reactant is weakly adsorbed component then less amount of product is produced due to the small residence time of the reactant and possible backward reaction, see Figure 5a,b. On the other hand, if reactant is strongly adsorbed then more product is produced due to large residence time of the reactant although backward reaction is also active, see Figure 5c,d. The increased reaction rate and conversion depict the expected rise in the temperature profile.

Figure 6 shows the results of a case study in which the sample is injected with a temperature, $T_{\text {inj }}$, differing from the inlet temperature of the mobile phase, $T_{\text {ref }}$. Here, we have chosen, in addition to $T_{\text {ref }}=300 \mathrm{~K}, T_{\mathrm{inj}}=290 \mathrm{~K}$ and $310 \mathrm{~K}$. As expected, the lower injection temperature has increased the retention time and vice versa. A significant dif- 
ference can be seen in the shapes and heights of concentration and temperature profiles. The results of these calculations are shown here to indicate the potential of the model and the solution technique to further optimize this hitherto hardly considered possibility of increasing specific performance parameters of separations.

Figure 7 shows the influence of the density times heat capacity ratio of solid to liquid phases $\left(\rho^{S} c_{p}^{S} / \rho^{L} c_{p}^{L}\right)$ on concentration and temperature profiles. This ratio effects the speeds of temperature and concentration profiles inside the column. For $\rho^{S} c_{p}^{S} / \rho^{L} c_{p}^{L}<1$ the temperature wave is moving faster than the concentration profiles (plots (a) and (b)). For $\rho^{S} c_{p}^{S} / \rho^{L} c_{p}^{L}=1$ both concentration and temperature profiles are moving at similar speeds (plots (c) and (d)). On the there hand, for $\rho^{S} c_{p}^{S} / \rho^{L} c_{p}^{L}>1$ the temperature wave is moving slowly as compared to the concentration profiles (plots (e) and (f)). Thus, concentration and temperature profile are coupled in the case of $\rho^{S} c_{p}^{S} / \rho^{L} c_{p}^{L}=1$ and are decoupled for $\rho^{S} c_{p}^{S} / \rho^{L} c_{p}^{L} \lessgtr 1$. It can be also seen from the plots of Figure 7 that temperature fluctuations are high in the coupling case as compared to the decoupling cases. Moreover, conversation of the reactant into products is high in the coupling case. Thus, analogous to gas-solid reactors, the ratio $\rho^{S} c_{p}^{S} / \rho^{L} c_{p}^{L}$ plays a very important role in non-isothermal reactive liquid chromatography.

Generally, the physical properties like densities, heat capacities and the transport coefficients depend on temperature. However, in the relative small temperature range identified to be relevant in liquid chromatography, these effects are small and indeed negligible. Thus, we have not considered the temperature dependencies of these parameters in the model equations.

\subsection{Four-component reaction $(A+B \rightleftarrows C+D)$}

This section considers four-component reactions describing as a concrete reaction the often studied hydrolysis of an ester, see e.g. Tien and Seidel-Morgenstern (2011). For this reversible reaction, the rate expression in Eq. (7) is replaced by

$$
r^{\text {het }}=k^{\text {het }}\left(T_{s}\right)\left(q_{A} q_{B}-\frac{q_{C} q_{D}}{K_{e q}^{\text {het }}\left(T_{s}\right)}\right),
$$


where, $q_{i}$ denotes the loading of component $i$ on the solid phase. The standard parameters used in this test problems are given in Table 3. In this reaction, the cheaper water (component $B$ ) is chosen as the carrier component and also to efficiently regenerate the column prior to the next feed injection (of $A$ ).

Figure 8 represents the hydrolysis of Methyl Formate A to Formic Acid and Methanol $(\mathrm{C}$ and $\mathrm{D})$, respectively. As the reaction is carried out in a large excess of water $(\mathrm{B})$, the concentration of water is assumed unchanged in the course of reaction. We have considered the adsorption equilibrium constants $a_{A}=0.888, a_{B}=1.0, a_{C}=0.643$ and $a_{D}=0.458$ at temperature $T^{\mathrm{ref}}=298 \mathrm{~K}$. This means that the reactant $A$ elutes between the two products $C$ and $D$. Moreover, a heterogeneous reaction rate constant $k^{\text {het }}=8.38 \times 10^{-3} \mathrm{~min}^{-1} \mathrm{~mol}^{-1} l$ and a reaction equilibrium constant $K_{\mathrm{eq}}^{\text {het }}=0.22$ are used in the numerical simulation. The figure shows the concentration profiles of the reactant and products for two different values of the mass transfer coefficient, i.e. $k=1 \mathrm{~min}^{-1}$ and $k=100 \mathrm{~min}^{-1}$. Although rise in the temperature is not much significant, adsorption and desorption peaks can be easily seen in the temperature profiles of Figure 8. The first sharp downward peak in thermal wave originates from the desorption of water from the resin, which corresponds to an endothermic process. The second upward peak is due to adsorption while the third downward peak arises again due to the desorption of water. Moreover, for small value of $k$ the process is still non-equilibrium and the diffusion phenomena dominates the convection process. Conversion and separation of components improves on increasing the magnitude of $k$.

The smaller temperature fluctuations in Figure 8 are due to the larger speed of the temperature wave compared to the concentration profiles, i.e. temperature wave is decoupled from the concentration profiles. However, the temperature rise can be enhanced by changing the values of $\rho^{S} c_{p}^{S}$ and $\rho^{L} c_{p}^{L}$.

Figure 9 shows the hydrolysis of Methyl Acetate. Methyl Acetate reacts with water to form Acetic Acid and Methanol. The Henry's constants for this case study are $a_{A}=1.044875$, $a_{B}=1.0, a_{C}=0.643438$ and $a_{D}=0.530564$ at reference temperature $T^{\text {ref }}=298 \mathrm{~K}$. Here, the heterogeneous reaction rate constant $k^{\text {het }}=3.4754 \times 10^{-4} \mathrm{~min}^{-1} \mathrm{~mol}^{-1} l$ and the 
reaction equilibrium constant $K_{\mathrm{eq}}^{\text {het }}=0.14$.

Figures 10 and 11 show the effects of different permutations of adsorption coefficients (Henry's constants) on the elution and temperature profiles. An increased conversion and a better separation is observed in Case 2 when Methyl Formate has low adsorption than Formic Acid.

In further case studies (not presented here), it was observed that changing the heat transfer coefficient $h_{p}$ (c.f. Eqs. (3) and (4)) has no significant impact on the results.

\section{Conclusion}

A non-isothermal and non-equilibrium model of reactive chromatography was formulated and numerically approximated. The model consists of a system of convection-diffusionreaction partial differential equations for concentrations and temperatures, coupled with differential and algebraic equations. A high resolution finite volume scheme was proposed to solve the model equations. A few case studies of reversible reactions with three and four components involved were considered and analyzed. The numerical case studies demonstrated the significant coupling of concentration and thermal fronts and identified key parameters that influence the reactor performance. The numerical results also verified the accuracy of proposed numerical algorithm. Results of additional calculations revealed some potential for selecting different temperatures for the mobile phase and the injected feed. It was found that, analogous to gas-solid reactors, the density times heat capacity ratio of solid to liquid phases plays a major role in non-isothermal reactive liquid chromatography. It was observed that conversion can be higher under the non-isothermal operation of the reactor as compared to the isothermal operation. The computed results are seen to be very useful for understanding the complex front propagation phenomena and to optimize experimental conditions.

Brandt, A. , Mann, G., Arlt, W., 1997. Temperature gradients in preparative highperformance liquid chromatography columns. J. Chromatogr. A, 769, 109-117.

Borren, T., Fricke, J., 2005. Chromatographic reactors in preparative chromatography of 
fine chemicals and pharmaceutical agents. H. Schmidt-Traub (ed). Wiley-VCH Verlag, Weinheim, 371-395.

Carta, G., 1988. Exact analytical solution of a mathematical model for chromatographic operations. Chem. Eng. Sci., 43, 2877-2883.

Eigenberger, G., Kolios, G., NieKen, U., 2007. Efficient reheating of a reverse-flow reformerAn experimental study. Chem. Eng. Sci., 62, 4825-4841.

Fricke, J., Schmidt-Traub, H., Kawase, M., 2005. Chromatographic reactor, Ullmann's Encyclopedia of Industrial Chemistry. Wiley-VCH Verlag, Weinheim.

Ganetsos,G., Barker, P.E., 1993. Preparative and production scale chromatography (vol. 61). Marcel Dekker, Inc., New York, 375-523.

Glöckler, B., Dieter, H., Eigenberger, G., NieKen, U., 2006. Efficient reheating of a reverseflow reformer-An experimental study. Chem. Eng. Sci., 62, 5638-5643.

Graça, N.S., Pais, L.S., Silva, V.M.T.M., Rodrigues, A.E., 2012. Thermal effects on the synthesis of 1,1-dibutoxyethane in a fixed-bed adsorptive reactor, Chem. Eng. Tech., 35, 1989-1997.

Graça, N.S., Pais, L.S., Silva, V.M.T.M., Rodrigues, A.E., 2012. Thermal effects on the synthesis of acetals in a simulated moving bed adsorptive reactor, Chem. Eng. J., 207208, 504-513.

Guiochon, G., 2002. Preparative liquid chromatography. J. Chromatogr. A, 965, 129-161.

Guiochon, G., Lin, B., 2003. Modeling for preparative chromatography, Academic Press.

Guiochon, G., Felinger, A., Shirazi, D.G., Katti, A.M., 2006. Fundamentals of preparative and nonlinear chromatography, 2nd ed. ELsevier Academic press, New York. 
Javeed, S., Qamar, S., Seidel-Morgenstern, A., Warnecke, G., 2011. Efficient and accurate numerical simulation of nonlinear chromatographic processes. J. Comput. \& Chem. Eng., 35, 2294-2305.

Javeed, S., Qamar, S., Seidel-Morgenstern, A., Warnecke, G., 2012. Parametric study of thermal effects in reactive liquid chromatography. Chem. Eng. J., 191, 426-440.

Koren, B., 1993. A robust upwind discretization method for advection, diffusion and source terms. In C. B. Vreugdenhil, B. Koren (Eds). Numerical Methods for Advection-Diffusion Problems. Volume 45 of Notes on Numerical Fluid Mechanics, 117-138, Vieweg, Braunschweig.

Kruglov, A., 1994. Methanol synthesis in a simulated countercurrent moving-bed adsorptive catalytic reactor. Chem. Eng. Sci., 49, 4699-4716.

Rhee, H.-K., Aris, R., Amundson, N.R., 1989. First-order partial differential equations. Theory and Application of Hyperbolic Systems of Quasilinear Equations, vol. II. Prentice-Hall, New Jersey.

Ruthven, D.M., 1984. Principles of adsorption and adsorption processes, WileyInterscience, New York.

Sainio, T., 2005. Ion-exchange resins as stationary phase in reactive chromatography. Acta Universitatis Lappeenrantaensis 218, Diss. Lappeenranta University of Technology, Finland.

Sainio, T., Kaspereit, M., Kienle, A., Seidel-Morgenstern, A., 2007. Thermal effects in reactive liquid chromatography. Chem. Eng. Sci., 62, 5674-5681.

Sainio, T., Zhang, L., Seidel-Morgenstern, A., 2011. Adiabatic operation of chromatographic fixed-bed reactors. Chem. Eng. J., 168, 861-871. 
Sardin, M., Schweich, D., Villermaux J., 1993. Preparative fixed-bed chromatographic reactor, Preparative and Production Scale Chromatography. G. Ganetsos, P.E. Barker (Eds). Marcel Dekker Inc., New York, USA, 477-522.

Sweby, P.K., 1984. High resolution schemes using flux limiters for hyperbolic conservation laws. SIAM J. Numer. Anal., 30, 995-1011.

Tien, Vu-D., Seidel-Morgenstern, A., 2011. Quantifying temperature and flow rate effects on the performance of a fixed-bed chromatographic reactor. J. Chromatogr. A, 1218, 8097-8109.

Villermaux, J., 1981. The chromatographic reactor in percolation processes: Theory and application. A.E. Rodrigues, D. Tondeur (Eds). Sijthoffen Noordhoff, Alpena an den Rijn, The Netherlands, 539-588.

Xiu, G., Li, P., Rodrigues, A.E., 2002. Sorption-enhanced reaction process with reactive regeneration. Chem. Eng. Sci., 57, 3893-3908.

Yongsunthon, I., Alpay, E., 1999. Design of periodic adsorptive reactors for the optimal integration of reaction, separation and heat exchange. Chem. Eng. Sci., 2647-2657.

Zhong, G.M., Meunier, F., 1994. Interference and heat effects: moment analysis for twocomponent chromatography. J. Chromatogr. A, 658, 355-360. 
Table 1: Parameters used for the three-component reactive elution $(A \rightleftarrows B+C)$ (c.f. Sainio (2007); Tien and Seidel-Morgenstern (2011))

\begin{tabular}{|c|c|c|c|}
\hline Description & Symbols & Value & Unit \\
\hline external porosity & $\epsilon$ & 0.4 & - \\
\hline column length & $L$ & 0.27 & $m$ \\
\hline time of injection & $t_{\text {inj }}$ & 16.67 & $\min$ \\
\hline interstitial velocity & $u$ & 0.00621738 & $m / \min$ \\
\hline concentration of reactant $\mathrm{A}$ & $c_{A}^{\text {inj }}$ & 3.0 & $\mathrm{~mol} / \mathrm{l}$ \\
\hline concentration of product B & $c_{B}^{\text {inj }}$ & 0.0 & $\mathrm{~mol} / \mathrm{l}$ \\
\hline concentration of product $\mathrm{C}$ & $c_{C}^{\mathrm{inj}}$ & 0.0 & $\mathrm{~mol} / \mathrm{l}$ \\
\hline constant in langmuir isotherm & $a_{A}$ & 1.0 & - \\
\hline constant in langmuir isotherm & $a_{B}$ & 0.7 & - \\
\hline constant in langmuir isotherm & $a_{C}$ & 1.3 & - \\
\hline density times heat capacity in liquid phase & $\rho^{L} C_{p}^{L}$ & 4.0 & $k J / K l$ \\
\hline density times heat capacity in solid phase & $\rho^{S} C_{p}^{S}$ & 4.0 & $k J / K l$ \\
\hline activation energy & $E_{A}$ & 60 & $k J / m o l$ \\
\hline axial dispersion coefficient & $D_{z}$ & $10^{-7}$ & $m^{2} / \min$ \\
\hline conductivity coefficient & $\lambda_{z}$ & $10^{-8}$ & $m^{2} k J / \operatorname{minl} K$ \\
\hline reference temperature & $T^{\mathrm{ref}}$ & 300 & $K$ \\
\hline heat transfer coefficient & $h_{p}$ & 24 & $k J / \operatorname{minm}^{2} K$ \\
\hline radius of solid particle & $R_{p}$ & $2.0 \times 10^{-6}$ & $m$ \\
\hline general gas constant & $R_{g}$ & 0.008314 & $k J / \operatorname{molK}$ \\
\hline reaction equilibrium constant & $K_{\text {eq }}^{\text {het }}$ & 2.0 & $\mathrm{~mol} / \mathrm{l}$ \\
\hline heterogeneous reaction rate constant & $k^{\text {het }}$ & 0.006 & $\min ^{-1}$ \\
\hline
\end{tabular}


Table 2: Three-component elution: Here $X_{A}[\%]=100 \times \frac{n_{A}^{\text {in }}-n_{A}^{\text {out }}}{n_{A}^{\text {in }}}$ and $k=1200 \mathrm{~min}^{-1}$.

\begin{tabular}{|c|cccccccc|}
\hline parameters & $\xi_{A}$ & $\xi_{B}$ & $\xi_{C}$ & $\sigma_{\xi, k}$ & $X_{A}$ & $\Delta H^{\text {out }}$ & $\Delta H_{\mathrm{err}}$ & $E_{\mathrm{H}}$ \\
\hline$[\mathrm{kJ} / \mathrm{mol}]$ & {$[\mathrm{mol}]$} & {$[\mathrm{mol}]$} & {$[\mathrm{mol}]$} & {$[\%]$} & {$[\%]$} & {$[\mathrm{kJ}]$} & {$[\mathrm{kJ}]$} & {$[\%]$} \\
\hline$\Delta H_{\mathrm{A}}=0, \Delta H_{\mathrm{R}}=0$ (isothermal) & 0.008 & 0.008 & 0.008 & 0.006 & 31 & 0.0 & - & - \\
$\Delta H_{\mathrm{A}}=-40, \Delta H_{\mathrm{R}}=0$ & 0.009 & 0.009 & 0.009 & 0.008 & 37 & 0.002 & - & - \\
$\Delta H_{\mathrm{A}}=-60, \Delta H_{\mathrm{R}}=0$ & 0.008 & 0.008 & 0.008 & 0.006 & 31 & 0.001 & - & - \\
$\Delta H_{\mathrm{A}}=-0, \Delta H_{\mathrm{R}}=-20, E_{\mathrm{A}}=60$ & 0.009 & 0.009 & 0.009 & 0.006 & 39 & 0.230 & -0.001 & 0.38 \\
$\Delta H_{\mathrm{A}}=-0, \Delta H_{\mathrm{R}}=-40, E_{\mathrm{A}}=60$ & 0.012 & 0.012 & 0.012 & 0.006 & 48 & 0.480 & -0.002 & 0.32 \\
$\Delta H_{\mathrm{A}}=-60, \Delta H_{\mathrm{R}}=-20, E_{\mathrm{A}}=60$ & 0.011 & 0.011 & 0.011 & 0.006 & 43 & 0.110 & -0.001 & 0.58 \\
$\Delta H_{\mathrm{A}}=-60, \Delta H_{\mathrm{R}}=-50, E_{\mathrm{A}}=60$ & 0.012 & 0.012 & 0.012 & 0.006 & 47 & 0.435 & $4.7 \mathrm{e}-4$ & 0.15 \\
$\Delta H_{\mathrm{A}}=-60, \Delta H_{\mathrm{R}}=-20, E_{\mathrm{A}}=100$ & 0.013 & 0.013 & 0.013 & 0.006 & 51 & 0.115 & -0.001 & 0.75 \\
\hline
\end{tabular}


Table 3: Parameters used for the four-component reactive elution: Hydrolysis of Methyl Formate to Methanol and Formic Acid $(A+B \rightleftarrows C+D)$ (c.f. Tien and Seidel-Morgenstern (2011))

\begin{tabular}{|c|c|c|c|}
\hline Description & Symbols & Value & Unit \\
\hline external porosity & $\epsilon$ & 0.216 & - \\
\hline column length & $L$ & 0.27 & $m$ \\
\hline interstitial velocity & $u$ & 0.083572 & $m / \min$ \\
\hline time of injection & $t_{\text {inj }}$ & 1.0 & $\min$ \\
\hline concentration of reactant $\mathrm{A}$ & $c_{A}^{\mathrm{inj}}$ & 3.0 & $\mathrm{~mol} / \mathrm{l}$ \\
\hline concentration of reactant $B$ & $c_{B}^{\text {inj }}$ & 0.0 & $\mathrm{~mol} / \mathrm{l}$ \\
\hline concentration of product $\mathrm{C}$ & $c_{C}^{\mathrm{inj}}$ & 0.0 & $\mathrm{~mol} / \mathrm{l}$ \\
\hline concentration of product D & $c_{D}^{\mathrm{inj}}$ & 0.0 & $\mathrm{~mol} / \mathrm{l}$ \\
\hline density times heat capacity in liquid phase & $\rho^{L} C_{p}^{L}$ & 4.0 & $k J / K l$ \\
\hline density times heat capacity in solid phase & $\rho^{S} C_{p}^{S}$ & 4.0 & $k J / K l$ \\
\hline axial dispersion coefficient & $D_{z}$ & $9.92510^{-6}$ & $m^{2} / \min$ \\
\hline conductivity coefficient & $\lambda_{z}$ & $10^{-6}$ & $m^{2} k J / \operatorname{minl} K$ \\
\hline activation energy & $E_{A}$ & 60 & $k J / m o l$ \\
\hline reference temperature & $T^{\mathrm{ref}}$ & 298 & $K$ \\
\hline heat transfer coefficient & $h_{p}$ & 24 & $k J / \operatorname{minm}^{2} K$ \\
\hline radius of solid particle & $R_{p}$ & $2.0 \times 10^{-6}$ & $m$ \\
\hline general gas constant & $R_{g}$ & 0.008314 & $k J / \operatorname{molK}$ \\
\hline
\end{tabular}



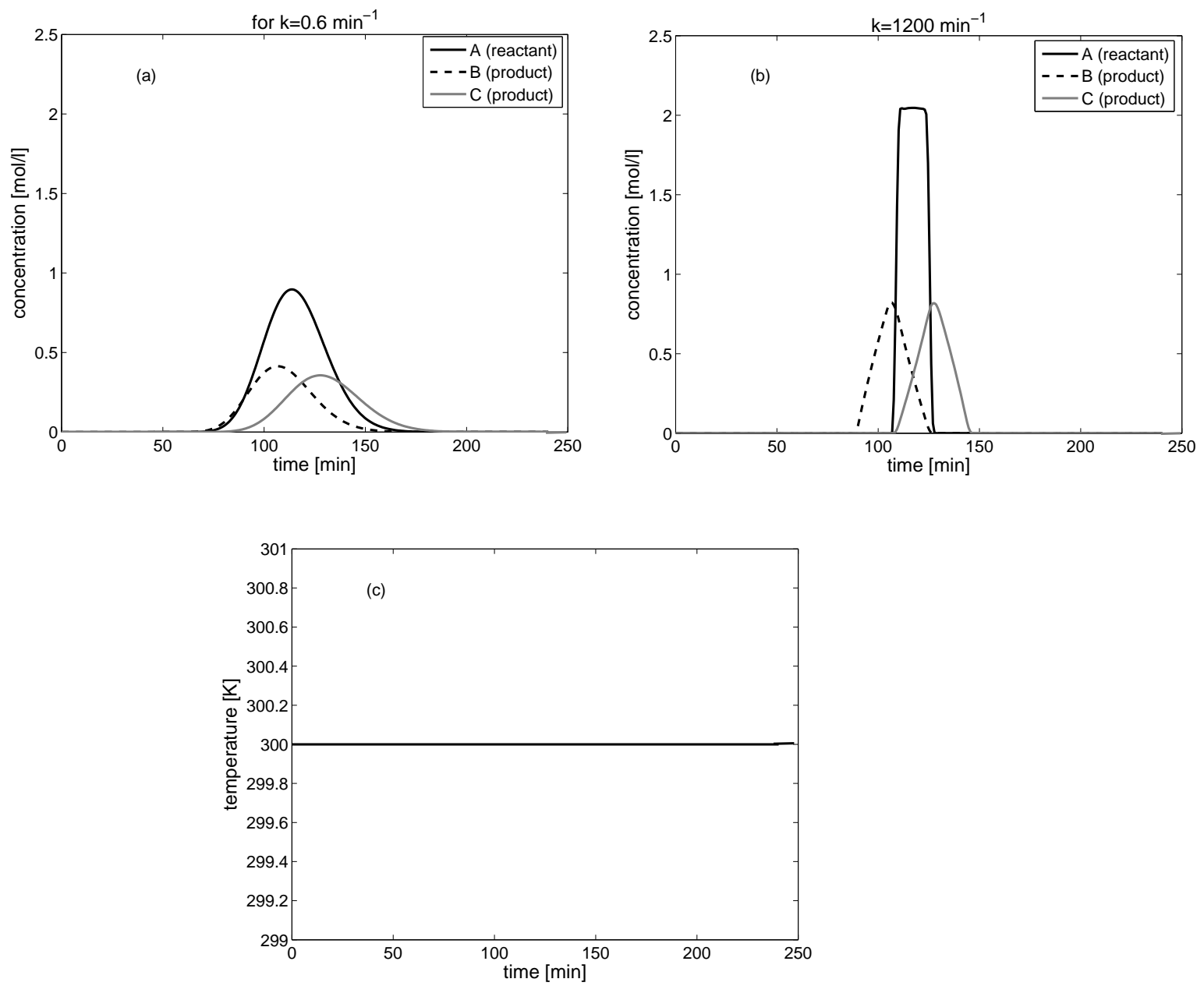

Figure 1: Three-component reaction (isothermal case): Concentration and temperature profiles for two different values mass transfer coefficient $k$. Here, $\Delta H_{A}=0=\Delta H_{R} \mathrm{~kJ} / \mathrm{mol}$. The values of other parameters are given in Table 1. 

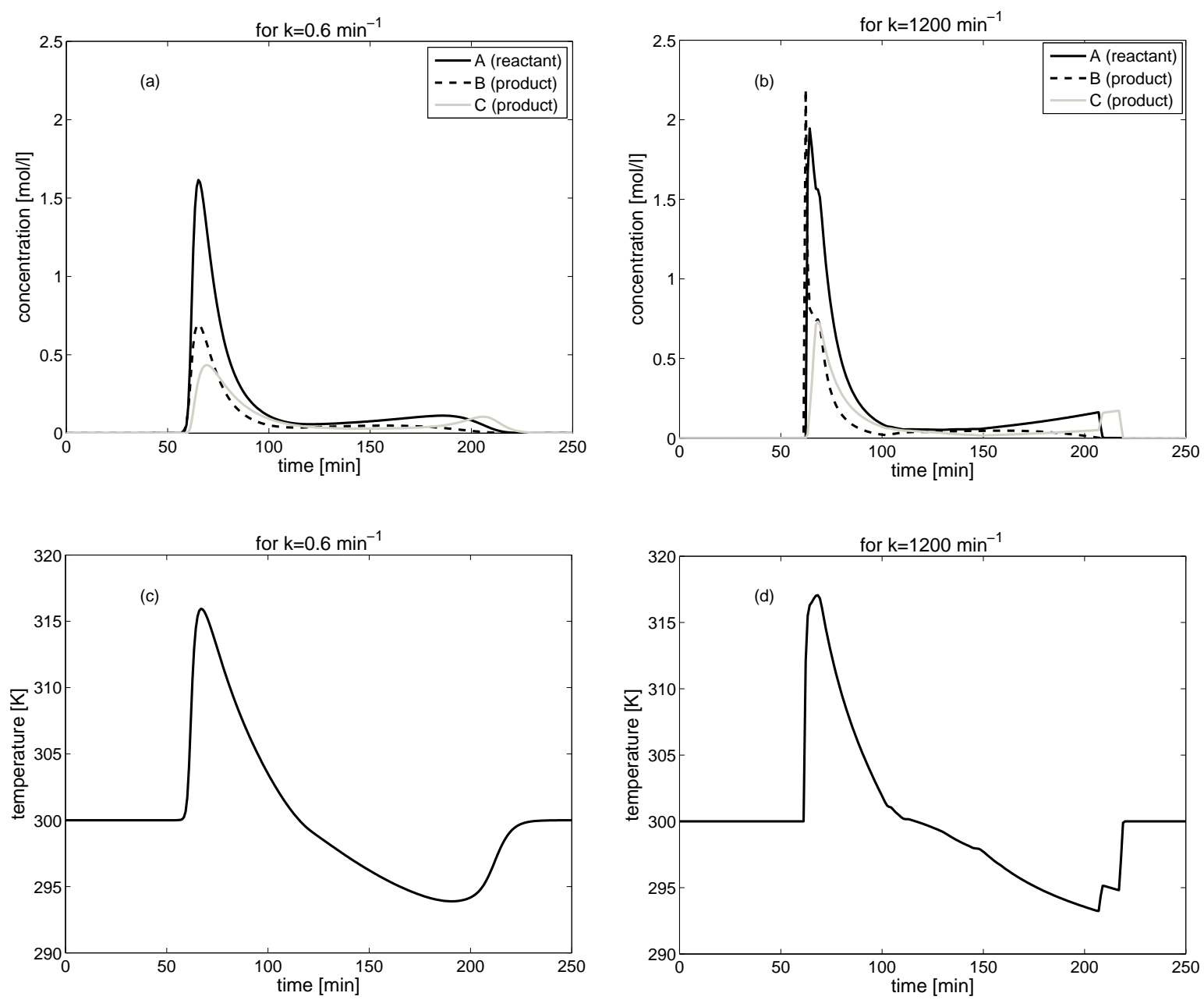

Figure 2: Three-component reaction: Influence of enthalpy of adsorption on concentration and temperature profiles for two different values mass transfer coefficient $k$. Here, $\Delta H_{R}=0 \mathrm{~kJ} / \mathrm{mol}$ and $\Delta H_{A}=-60 \mathrm{~kJ} / \mathrm{mol}$. The values of other parameters are given in Table 1. 

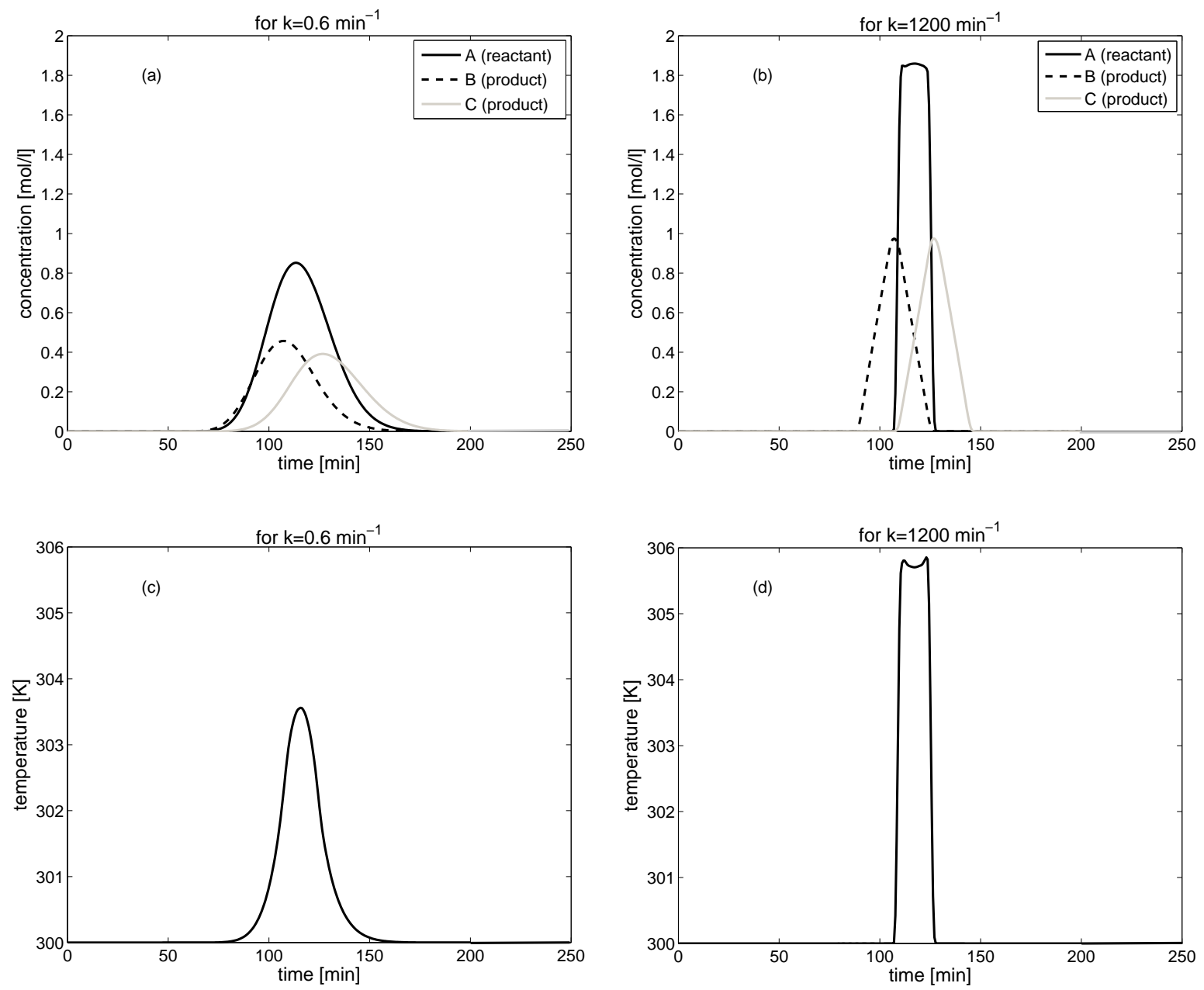

Figure 3: Three-component reaction: Influence of enthalpy of reaction on concentration and temperature profiles for two different values of mass transfer coefficient $k$. Here, $\Delta H_{R}=-20 \mathrm{~kJ} / \mathrm{mol}$ and $\Delta H_{A}=$ $0 \mathrm{~kJ} / \mathrm{mol}$. The values of other parameters are given in Table 1. 

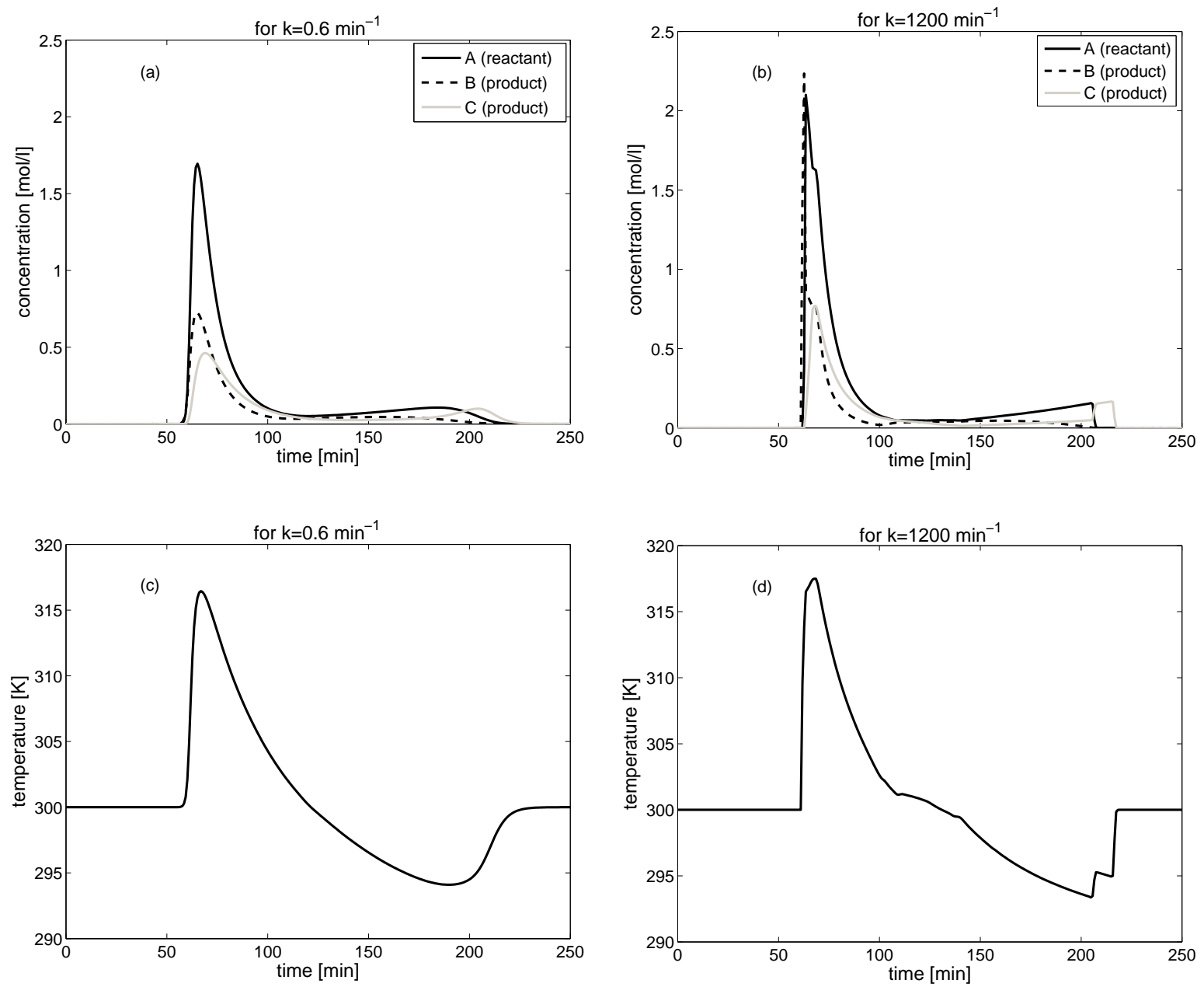

Figure 4: Three-component reaction: Influence of both enthalpies of reaction and adsorption on concentration and temperature profiles for two different values of mass transfer coefficient $k$. Here, $\Delta H_{R}=$ $-20 \mathrm{~kJ} / \mathrm{mol}$ and $\Delta H_{A}=-60 \mathrm{~kJ} / \mathrm{mol}$. The values of other parameters are given in Table 1 . 

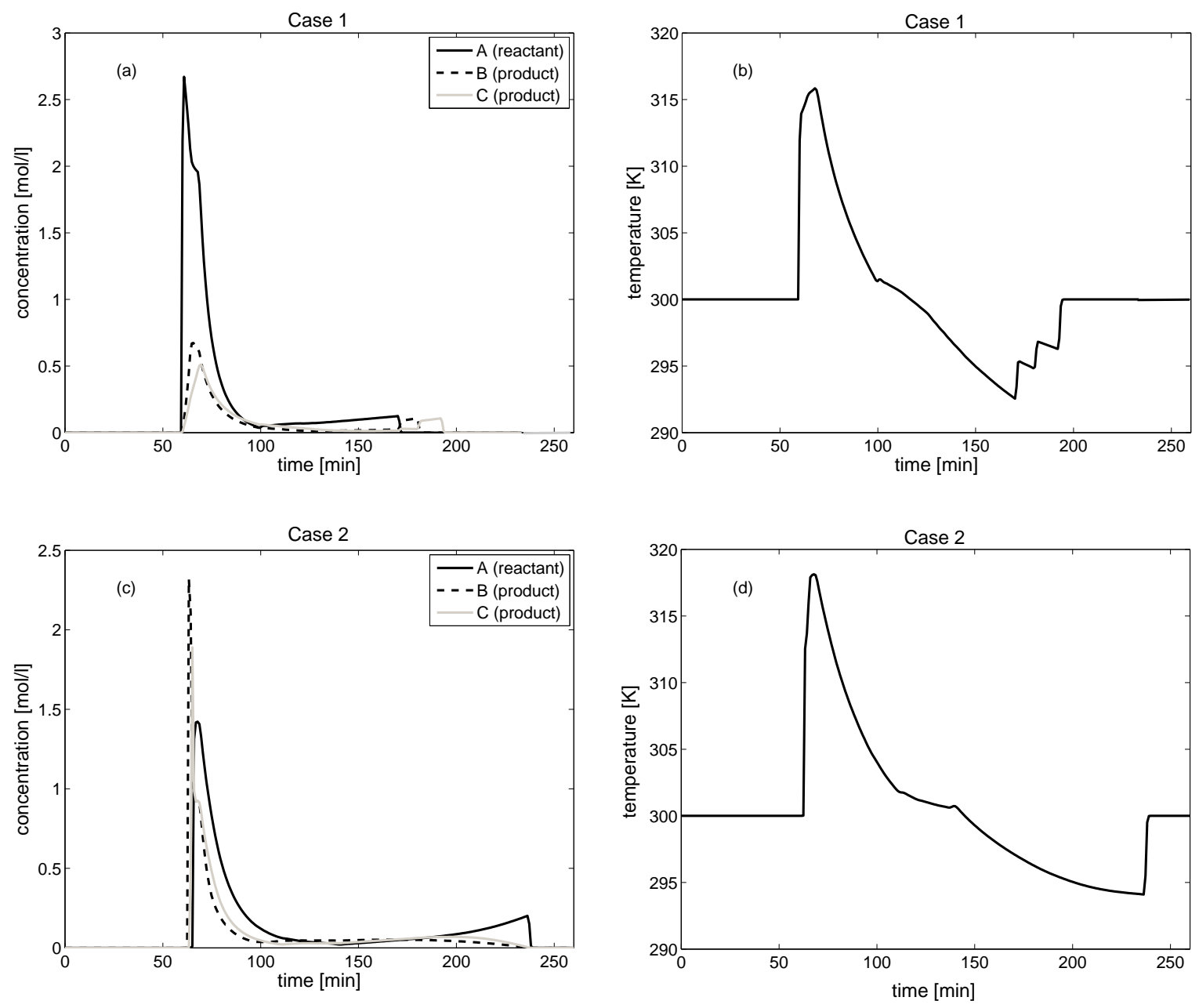

Figure 5: Three-component reaction: Influence of both enthalpies of reaction and adsorption, $\Delta H_{R}=$ $-20 \mathrm{~kJ} / \mathrm{mol}, \Delta H_{A}=-60 \mathrm{~kJ} / \mathrm{mol}$ with different permutation of adsorption coefficients. Here, $k=$ $1200 \mathrm{~min}^{-1}$. Case 1: $a_{A}=0.7, a_{B}=1.0, a_{C}=1.3$; Case $2: a_{A}=1.3, a_{B}=0.7, a_{C}=1.0$. The values of other parameters are given in Table 1 . 

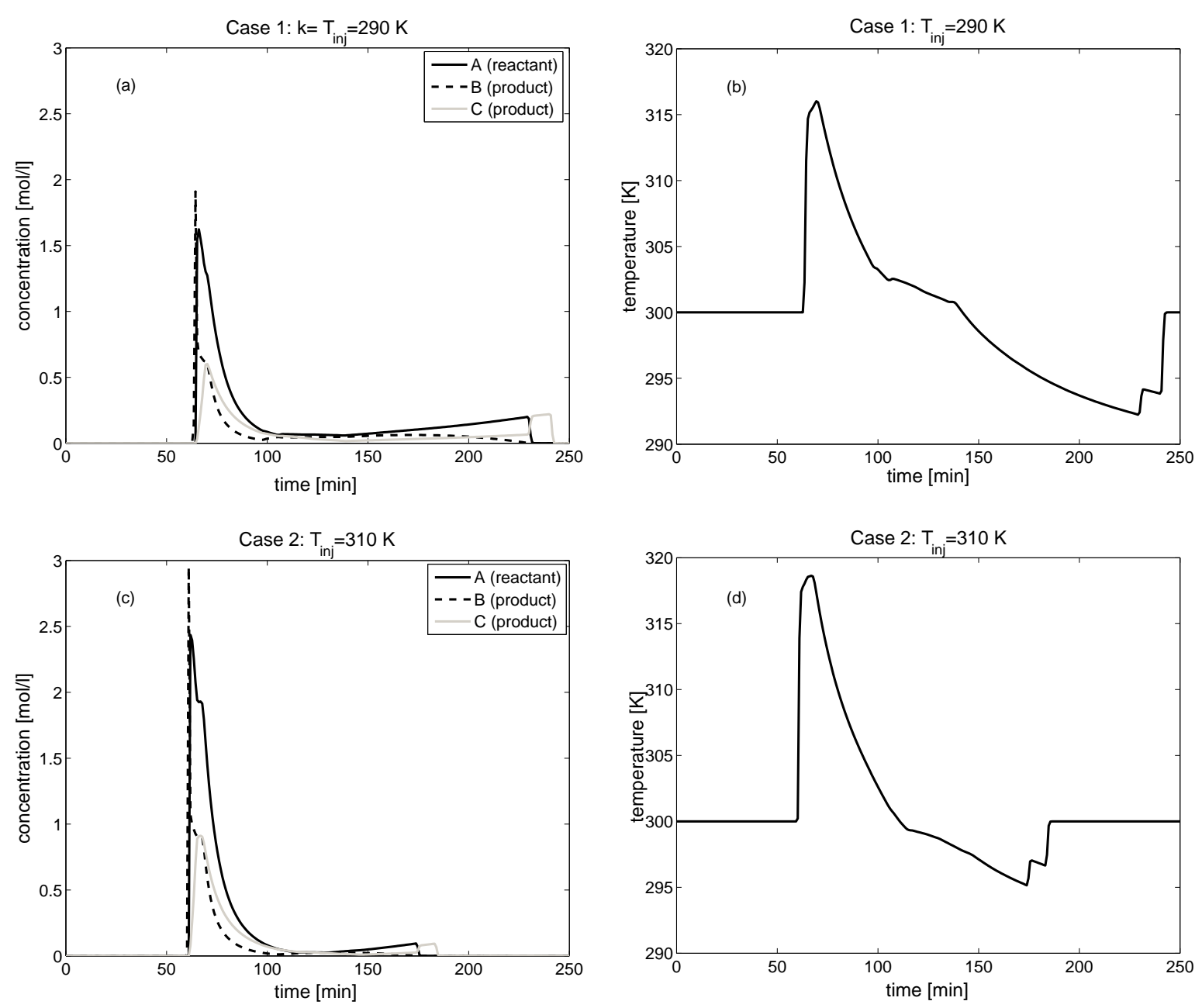

Figure 6: Three-component reaction: Influence of inlet temperature. Case 1: $T_{\text {inj }}=290 \mathrm{~K}$; Case 2: $T_{\mathrm{inj}}=310 \mathrm{~K}$. Here, $\Delta H_{R}=-20 \mathrm{~kJ} / \mathrm{mol}, \Delta H_{A}=-60 \mathrm{~kJ} / \mathrm{mol}$, and $\mathrm{k}=1200 \mathrm{~min}^{-1}$. The values of other parameters are given in Table 1. 

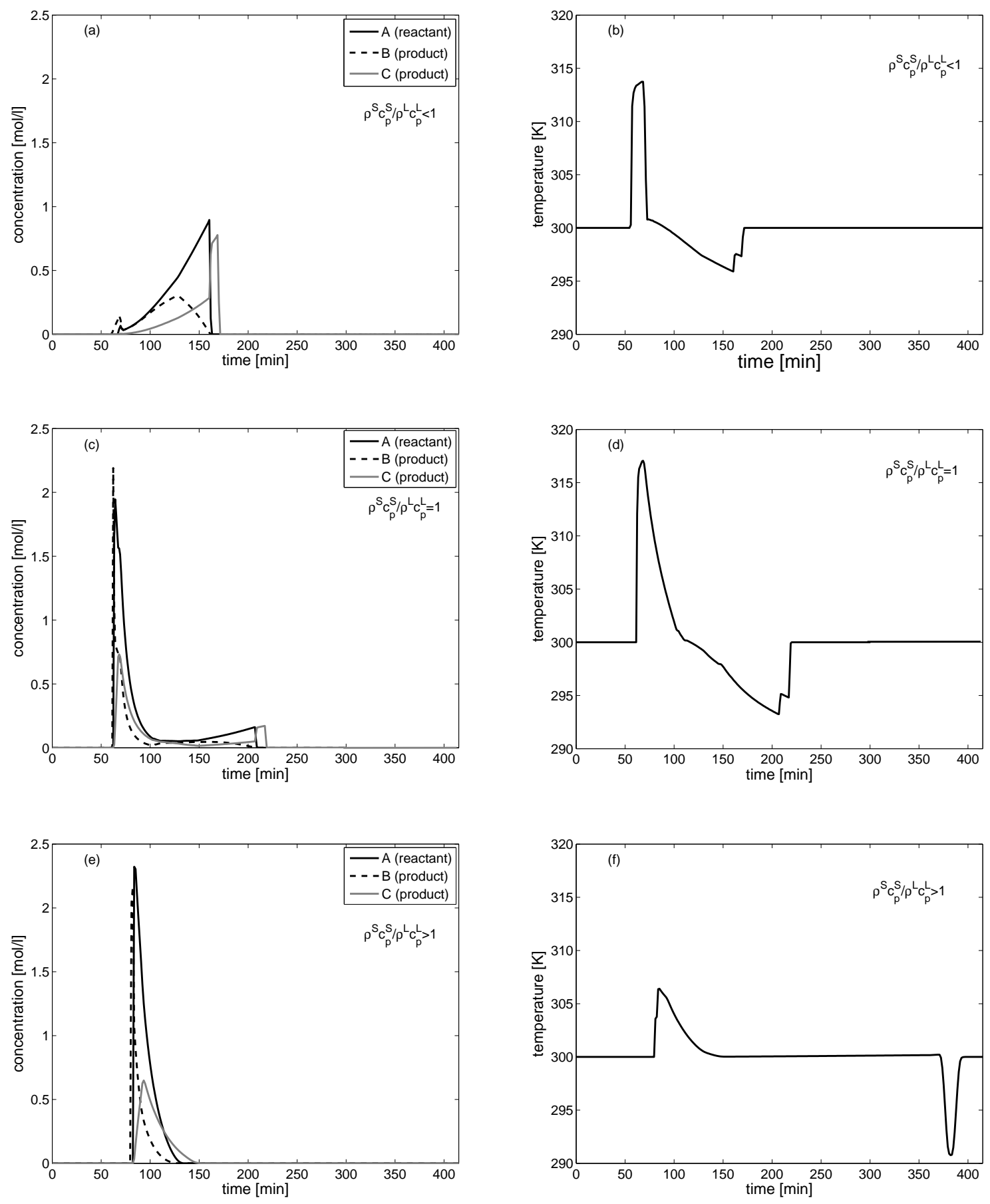

Figure 7: Three-component reaction: Influence of $\rho^{S} c_{p}^{S} / \rho^{L} c_{p}^{L}$ on concentration and temperature profiles. Plots (a)\&(b): $\rho^{S} c_{p}^{S}=4 k J / K l$ and $\rho^{L} c_{p}^{L}=20 k J / K l$; plots (c)\&(d): $\rho^{S} c_{p}^{S}=4 k J / K l$ and $\rho^{L} c_{p}^{L}=$ $4 k J / K l$; plots (e)\&(f): $\rho^{S} c_{p}^{S}=20 k J / K l$ and $\rho^{L} c_{p}^{L}=4 k J / K l$. Here, $\Delta H_{R}=-20 k J / m o l, \Delta H_{A}=$ $-60 \mathrm{~kJ} / \mathrm{mol}$, and $k=1200 \mathrm{~min}^{-1}$. The values of other parameters are given in Table 1. 

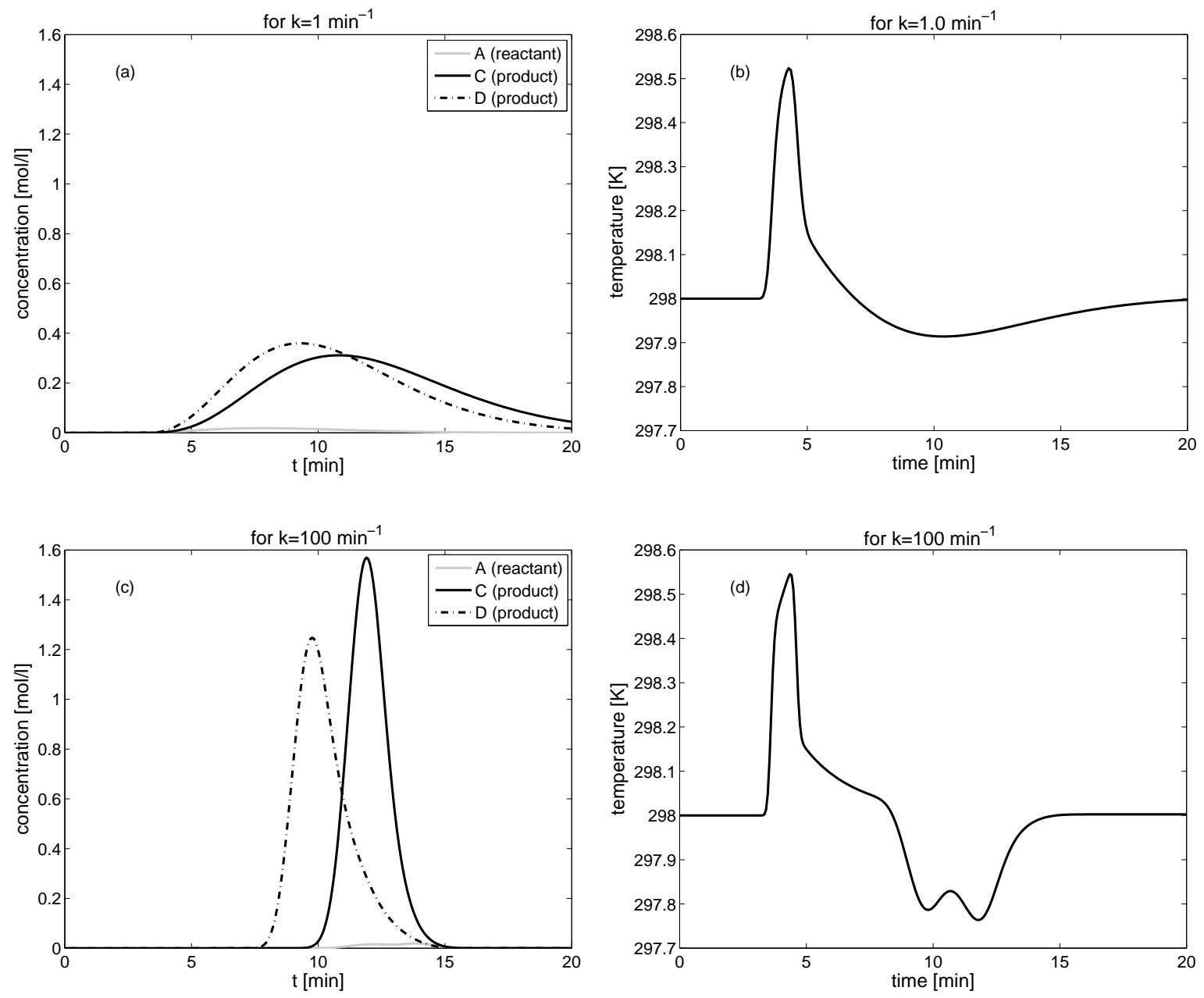

Figure 8: Four-component reaction: Hydrolysis of Methyl Formate. Here, $\Delta H_{A}=-40 \mathrm{~kJ} / \mathrm{mol}$ and $\Delta H_{R}=-20 \mathrm{~kJ} / \mathrm{mol}$. The values of other parameters are given in Table 3 . 

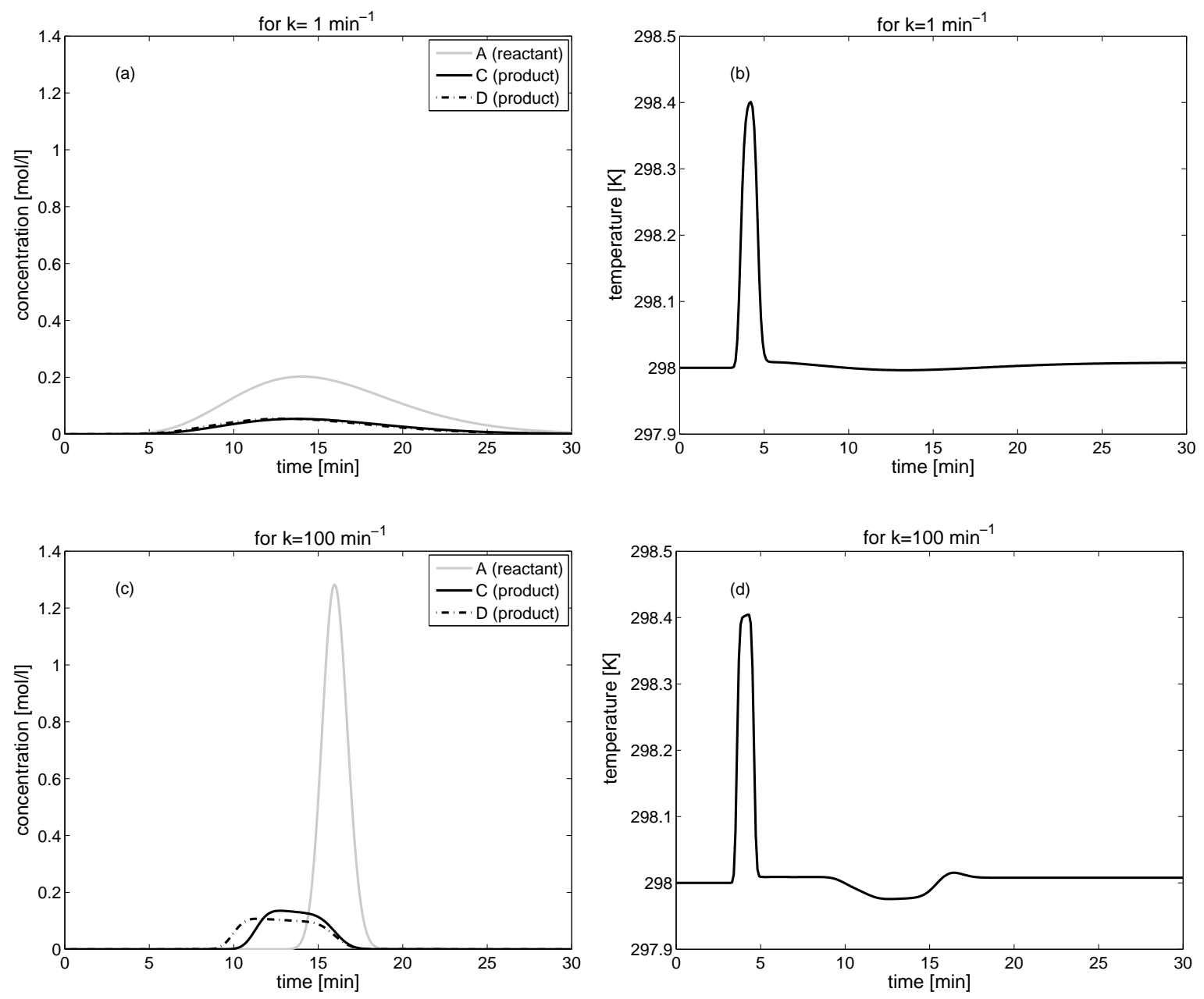

Figure 9: Four-component reaction: Hydrolysis of Methyl Acetate. Here, $\Delta H_{A}=-40 \mathrm{~kJ} / \mathrm{mol}$ and $\Delta H_{R}=-20 \mathrm{~kJ} / \mathrm{mol}$. The values of other parameters are given in Table 3 . 

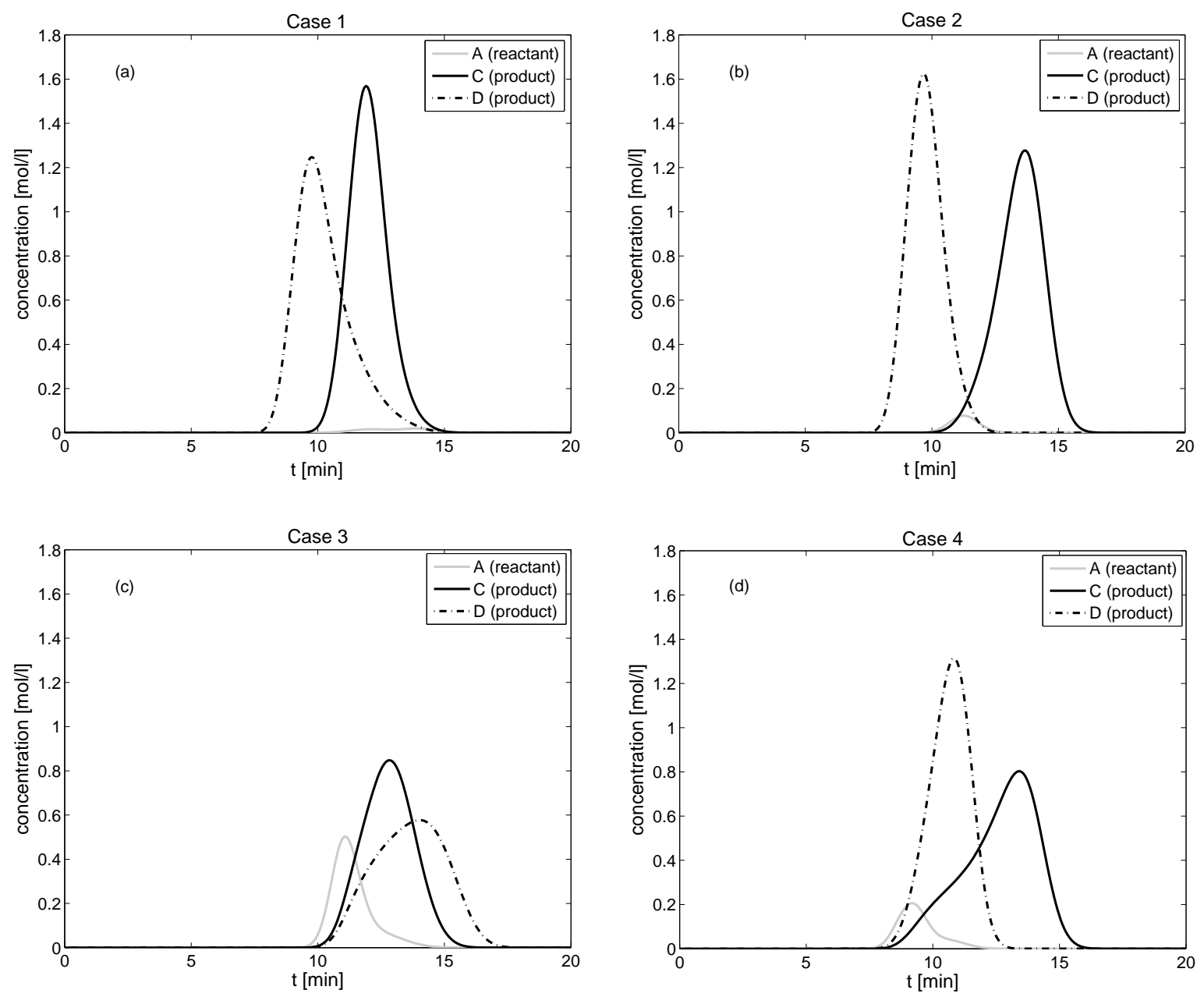

Figure 10: Four-component reaction: Effects of different permutations of adsorption coefficients on eultion profiles for the hydrolysis of Methyl Formate with $\Delta H_{A}=-40 \mathrm{~kJ} / \mathrm{mol}, \Delta H_{R}=-20 \mathrm{~kJ} / \mathrm{mol}$ and $\mathrm{k}=100$ $\min ^{-1}$. Case 1: $a_{A}=0.888, a_{B}=1.0, a_{C}=0.643, a_{D}=0.458 ;$ Case 2: $a_{A}=0.643, a_{B}=1.0, a_{C}=0.888$, $a_{D}=0.458 ;$ Case 3: $a_{A}=0.643, a_{B}=0.458, a_{C}=0.888, a_{D}=1.0 ;$ Case $4: a_{A}=0.458, a_{B}=1.0$, $a_{C}=0.888, a_{D}=0.643$. The values of other parameters are reported in Table 3. 

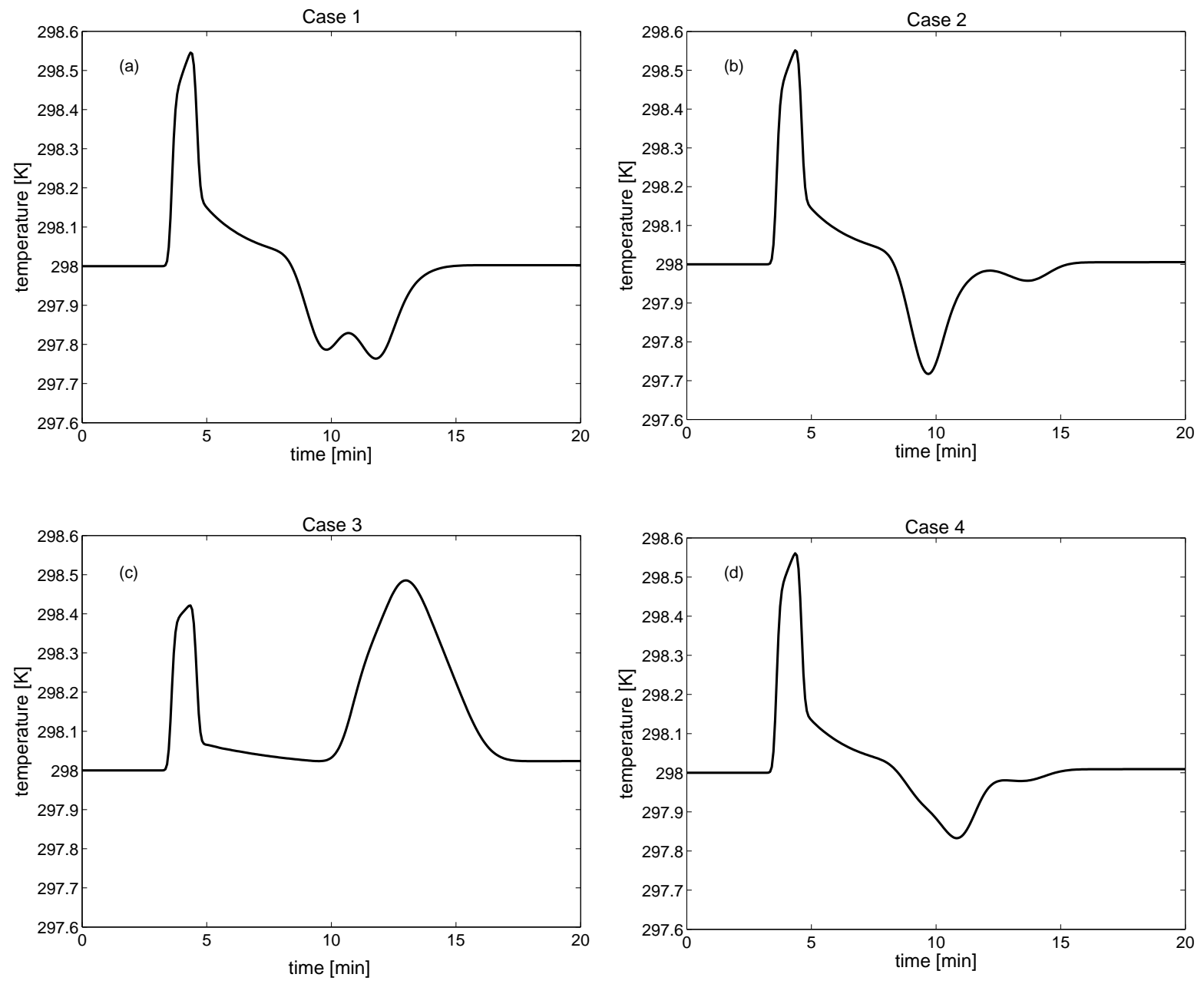

Figure 11: Four-component reaction: Effect of different permutation of adsorption coefficients on temperature profiles for the hydrolysis of methyl formate with $\Delta H_{A}=-40 \mathrm{~kJ} / \mathrm{mol}, \Delta H_{R}=-20 \mathrm{~kJ} / \mathrm{mol}$ and $k=100 \mathrm{~min}^{-1}$. Case 1: $a_{A}=0.888, a_{B}=1.0, a_{C}=0.643, a_{D}=0.458 ;$ Case 2: $a_{A}=0.643, a_{B}=1.0$, $a_{C}=0.888, a_{D}=0.458 ;$ Case 3: $a_{A}=0.643, a_{B}=0.458, a_{C}=0.888, a_{D}=1.0 ;$ Case 4: $a_{A}=0.458$, $a_{B}=1.0, a_{C}=0.888, a_{D}=0.643$. The values of other parameters are given in Table 3 . 